\title{
Eine NEAT-Grossbaustelle und ihre sozioökonomischen Auswirkungen auf die Standortregion
}

\author{
Simone Strauf, Manfred Walser, St. Gallen
}

\section{Einleitung}

Der Tunnelbau hat in der Schweiz eine lange Tradition und besondere Bedeutung. Mit dem Bau der grossen Alpentransversalen am Gotthard, Simplon und Lötschberg begann vor mehr als 100 Jahren die Verkehrseinbindung der Schweiz in Europa. Die Gotthardbahn als direkte Verbindung zwischen Deutschland und Italien war ein wichtiger Faktor für die technische und wirtschaftliche Entwicklung Europas im ausgehenden 19. und im 20. Jahrhundert. Mit der derzeit im Bau befindlichen Neuen Eisenbahn-Alpentransversalen (NEAT) integriert sich die Schweiz in das europäische Hochgeschwindigkeitsnetz. Herzstück der NEAT ist der neue Gotthard-Basistunnel, der mit einer Länge von $57 \mathrm{~km}$ der längste Eisenbahntunnel der Welt ist.

Wie schon der Bau der Alpentransversalen vor über 100 Jahren stellt auch der Bau der NEAT mit den beiden technisch höchst anspruchsvollen Tunnelprojekten Gotthard und Lötschberg eine neue Dimension des Bahnbaus dar. Die Baumassnahmen konfrontieren auch die angrenzenden Gemeinden mit neuen Herausforderungen, da von der Baustelle vielfältige Effekte auf die Gemeindeentwicklung ausgehen. Die historischen Baumassnahmen brachten spürbare Veränderungen in der Bevölkerungsstruktur mit sich, da während der jahre- bzw. jahrzehntelangen Dauer der Baumassnahmen zahlreiche Arbeitskräfte von ausserhalb in die Dörfer kamen und dort teilweise geblieben sind. Im Zusammenhang mit den TunnelJubiläen wurden verschiedentlich die Auswirkungen der Baustellen auf ihre jeweiligen Standortgemeinden im historischen Kontext untersucht. $\mathrm{Zu}$ nennen sind hier insbesondere die verschiedenen Arbeiten, die aus Anlass des 125-jährigen Jubiläums der Gotthardbahn erschienen sind (vgl. ViaStoria \& ElSASSER 2007), oder die im Jahr 2003 erschienene Dokumentation über das «Tripoli» in Grenchen, in der das Wohnen und Leben mit italienischen Tunnelbauern dargestellt wurde (vgl. Kultur-Historisches Museum Grenchen 2003). Im Jahr 2007 fand unter dem Titel «Cantina Transalpina» an sechs Orten entlang wichtiger Verkehrsachsen eine Ausstellung zur Alltagsgeschichte des Tunnelbaus statt (http://www.cantina-transalpina.ch). Bei all diesen wissenschaftlichen Arbeiten handelt es sich um retrospektive Arbeiten, die die Auswirkungen von grossen Baumassnahmen im historischen Kontext analysieren.
Im Zusammenhang mit dem Bau des neuen Gotthard-Basistunnels ergab sich nun die Chance, die Auswirkungen eines derartigen Jahrhundertbauwerks auf eine einzelne Gemeinde und Region im Rahmen einer Langzeitstudie zu analysieren. Am Beispiel der NEAT-Baustelle in Sedrun (Graubünden) wird untersucht, welche Effekte die Grossbaustelle auf die Gemeinde Tujetsch und die Obere Surselva hat, und welchen dauerhaften Nutzen sie für die Gemeinde erbringt. Dabei dient die Studie einerseits als Dokumentation und andererseits als Frühwarnsystem, um einschneidende Veränderungen frühzeitig zu erkennen und rechtzeitig darauf reagieren zu können. Auftraggeber der Studie sind die Gemeinde Tujetsch, der Kanton Graubünden und das Bundesamt für Verkehr (BAV), durchgeführt wird sie am Institut für Öffentliche Dienstleistungen und Tourismus (IDT-HSG) der Universität St. Gallen.

Mit dem dritten Zwischenbericht 2006 liegen nun Erfahrungen aus zehn Jahren Baustellenpraxis vor. Die Auswirkungen der Baumassnahme auf die Gemeinde werden im Folgenden nach einer kurzen Beschreibung der Forschungsmethodik anhand ausgewählter sozioökonomischer Kriterien dargestellt. Abschliessend wird versucht, Unterschiede und Gemeinsamkeiten zwischen früheren Tunnelbaustellen und der aktuellen NEAT-Baustelle in Sedrun in Bezug auf ihre regionalen Auswirkungen zu identifizieren.

\section{Sozioökonomische Auswirkungen von Grossbau- stellen früher und heute}

Früher wie heute lassen sich Auswirkungen der Baustellen auf ihre Standortgemeinde und -region identifizieren. Die Präsenz einer Grossbaustelle hinterlässt in einer Gemeinde Spuren, die teilweise sehr lange nachwirken. Während der ersten Hochphase des Tunnelbaus Ende des 19. und Anfang des 20. Jahrhunderts wurden zusätzliche Arbeitskräfte für den Tunnelbau benötigt. Sie kamen grossenteils aus Norditalien. Doch oft kamen sie nicht alleine, sondern brachten ihre Frauen und Kinder mit. In vielen Gemeinden wuchs die Zahl der Bewohner schnell an, im Jahr 1880 stieg beispielsweise die Bevölkerungszahl in der Gemeinde Göschenen von 300 auf 3'500 (vgl. CANtina Transalpina 2007). Für die Tunnelarbeiter und ihre Familien wurden Wohnbaracken errichtet, nicht selten entstand ein eigenes Quartier am Ortsrand der Gemeinde. Dieses Quartier war einerseits Lebens- 
raum für die zugewanderte Bevölkerung und andererseits eine Attraktion für die einheimische Bevölkerung. Die Lebensgewohnheiten der Mineursfamilien waren oft andere, die einheimische Bevölkerung war nicht nur mit ungewohnten Konsum-, Essens- und Lebensgewohnheiten konfrontiert, sondern auch die Sprache war fremd. Die Einheimischen schätzten zwar das neue und teilweise auch aufregende Unbekannte, doch taten sie sich hiermit teilweise auch schwer (vgl. Cantina Transalpina 2007).

Die Baustellen und die Tunnelarbeiter waren nicht nur eine Attraktion für die einheimische Bevölkerung, sondern auch für Touristen. So profitierte z.B. Kandersteg bereits Anfang des 20. Jahrhunderts beim Bau des Lötschbergtunnels von der Baustelle, die neugierige Reisende anzog (vgl. Cantina Transalpina 2007). Auch wirtschaftlich profitierten die Gemeinden von der Baustelle: in der Nähe der Quartiere der Tunnelarbeiter wurden Restaurants und kleine Geschäfte eröffnet, die zwar überwiegend von den Arbeitern, aber auch von den Einheimischen besucht wurden (vgl. Kultur-Historisches Museum Grenchen 2003). Die anfängliche Euphorie währte teilweise jedoch nicht lange, bereits nach einigen Monaten war das Barackendorf Tripoli in Grenchen nicht mehr interessant, und ein Grossteil der erst kurz vorher eröffneten Gaststätten musste wieder schliessen (FASNACHT 2003: 13). Ausgehend von der Baustelle und der hierfür benötigten Infrastruktur wurden teilweise auch Investitionen über die Bauzeit hinaus getätigt. So wurde beispielsweise das für die Tunnelbauarbeiter errichtete kleine Spital in Grenchen später als erstes öffentliches Spital der Gemeinde genutzt (vgl. WALTER 2003).

Es stellt sich nun die Frage, ob die Auswirkungen einer solchen Grossbaustelle mit jenen in heutiger Zeit vergleichbar sind.

\section{Methodisches Vorgehen der aktuellen Studie zur NEAT-Baustelle Sedrun}

Ausgangspunkt für die Studie war die einmalige Gelegenheit, innerhalb eines übersichtlichen Raumes die lokalen und regionalen Auswirkungen einer solchen Baumassnahme in einer Längsschnittanalyse zu erfassen, auszuwerten und zu dokumentieren. Für die Begleitforschung wurde ein transdisziplinärer Forschungsansatz gewählt, bei dem schon bei der Formulierung der zentralen Fragestellungen und bei der Auswahl der Kriterien und Indikatoren eng mit wichtigen Akteuren zusammengearbeitet wurde.

Als normativer Ansatz zur Bewertung dieser Auswirkungen wurde das Konzept der nachhaltigen Entwicklung verwendet, d.h. es sollten gleichermassen die sozialen, die wirtschaftlichen und die ökologischen Auswirkungen der Baustelle untersucht werden. In einem ersten Schritt wurde anhand einer Literaturanalyse zu Nachhaltigkeitsindikatoren und zur Prozessbewertung sowie zu derartigen Grossbaustellen ein umfangreiches Indikatorenset erarbeitet (Abb.1).

Mit Vertretern der Gemeinde, der Auftraggeber und weiterer Interessensgruppen wurden aus diesem umfangreichen Kriterien- und Indikatorenkatalog 13 Kernindikatoren für die Bewertung der feststellbaren Auswirkungen entwickelt, denen besonderes Augenmerk gelten sollte. Im Mittelpunkt standen dabei die Aussagekraft aus Sicht der Akteure, die Relevanz für die Gesamtauswirkungen aus Sicht einer nachhaltigen Entwicklung sowie die Messbarkeit (siehe Abb. 2).

Der ausführliche Kriterienkatalog wird in einem Drei-Jahres-Rhythmus erhoben und in einem Bericht zusammengefasst. Datengrundlagen bilden einerseits Sekundärstatistiken der Gemeinde Tujetsch und des Kantons Graubünden sowie Auswertungen der Alptransit Gotthard AG als Bauherrin. Wiederholt wurden auch empirische Daten erhoben, z.B. im Rahmen einer Unternehmensbefragung (2000), einer Strassenumfrage in der Gemeinde (2000), einer Befragung der Baustellenbeschäftigten (2004) sowie bei Gemeindeversammlungen $(2003,2007)$. Die Ergebnisse werden laufend öffentlich vorgestellt und diskutiert.

\section{Die NEAT-Baustelle in Sedrun}

\subsection{Ausgangslage}

Die Erschliessung des Gotthard-Basistunnels erfolgt über fünf Zugänge: Die Eingangsportale befinden sich in Erstfeld im Norden und Bodio im Süden, dazwischen liegen die Teilabschnitte Amsteg, Sedrun und Faido (vgl. Abb. 3). Der Teilabschnitt Sedrun wird über einen $1 \mathrm{~km}$ langen Zugangsstollen und zwei $800 \mathrm{~m}$ tiefe Schächte erschlossen. In diesem Teilabschnitt wird auch eine der beiden Multifunktionsstellen gebaut, welche in der Betriebsphase bahntechnische Einrichtungen, aber auch Nothaltestellen und Spurwechsel beherbergen sollen. Der Ausbau der Nothaltestelle zu einem permanenten regulären Bahnhof wird immer noch als «Vision Porta Alpina» diskutiert.

Mit der Wahl Sedruns als Standort für den Zwischenangriff und die Multifunktionsstelle des GotthardBasistunnels ändern sich für die Gemeinde die Rahmenbedingungen für einen Zeitraum von etwa 20 Jahren.

\subsection{Wirtschaftliche Auswirkungen}

Sowohl die unternehmerischen Aktivitäten der Alptransit Gotthard AG als Bauherrin und ihrer Auftragneh- 


\section{Bereich Wirtschaft}

Versorgungsstrukturen (Preisentwicklung verschiedener Güter und Dienstleistungen)

Tourismusentwicklung (Baustellen-Besucher, Logiernächte, Wertschöpfung, FerienwohnungsBesatz, touristische Infrastruktur)

Wirtschaftsstruktur (Umsätze und Arbeitsplätze in ausgewählten Unternehmen, Entwicklung und Umsätze im Tourismus, Unternehmensgründungen, Innovation, Absatz lokaler/regionaler Produkte und Dienstleistungen)

Handlungsfähigkeit der Gemeinde (Steuern und Abgaben, Gemeindefinanzen und Investitionen)

\section{\begin{tabular}{|c|c|}
\hline Bereich Soziales & $\begin{array}{c}\text { Obere } \\
\text { Surselva }\end{array}$ \\
Stimmung in der Orts- und Talbevölkerung &
\end{tabular}}

\section{nd}

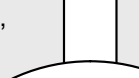
Flächenbilanz, Entwicklung von Wohnflächen,
landwirtschaftlich genutzten Flächen und Waldflächen, Materialablagerungen, Hangrutschungen/Lawinen-

abgänge, Baumaterialreserven)

Umweltgerechte Baumassnahmen (Umweltaudits, Materialverbrauch/Recycling, Nachnutzung Infrastruktur, ökologische Sensibilisierung der Arbeiterschaft, Ökologie im Besucherinformationszentrum, Verwendung gefährlicher Materialien, Feuerwehreinsätze, Schadstoffaustrag von Ablagerungen, Schutz besonderer Lebensräume)

(Anfragen und Beschwerden, Bevölkerung, Nettomigrationsrate, Stimmung allgemein in Umfragen) Integration der Bauarbeiter (Administratives, Bedürfnisse wie z.B. Religionsausübung, Waren- und Freizeitangebot, Kultur und Vereine, Wohnverhältnisse, Familienstrukturen, Integrationsleistung der öffentlichen Hand, Arbeitsbedingungen, medizinische Versorgung, Unfälle)

Belästigungen durch die Baustelle (Lärm-, Staubemissionen, Verkehrsbelastungen)

Verhältnis Gemeindepolitik - Bevölkerung (Informiertheit, Einbeziehung von Nichtregierungsorganisationen, Informationsfluss Alptransit - Administrationen, politische Stimmung)

Abb. 1: Mögliche lokale und regionale Auswirkungen einer Grossbaustelle aus Sicht einer nachhaltigen Entwicklung

Possible local and regional impacts of a big construction site based on the concept of sustainable development Effets locaux et régionaux possibles d'un gros chantier en vue d'un développement durable

mer als auch die Ausgaben der Baustellenbeschäftigten haben Einfluss auf die lokale und regionale Wirtschaft. Eine Studie zu den regionalwirtschaftlichen Effekten der NEAT-Baustelle auf die Region Obere Surselva beziffert die zusätzlichen Umsätze in der Region durch die Baustelle für die Zeit von 1996 bis 2003 auf 122 Mio CHF (vgl. Strauf, Walser \& Bieger 2004). Die beteiligten Unternehmen vergeben Aufträge an lokale und regionale Auftragnehmer, und die Beschäftigten tätigen Ausgaben in der Gastronomie und im Einzelhandel (auch wenn der Grossteil ihres Einkommens zu den Familien in die Heimatländer abfliesst).

In den ersten drei Jahren waren nur etwa 100 Personen auf der Baustelle beschäftigt. Seit 2002 hat sich die Zahl der Baustellenbeschäftigten kontinuierlich erhöht und wird 2007 maximal 700 Personen umfassen, ab 2008 bis Ende der Bauzeit werden es sukzessive weniger sein. In der Regel handelt es sich bei den Beschäftigten um Spezialisten, die als Ingenieure und Mineure ein paar Jahre auf der Baustelle verbringen und dann an ihren nächsten Einsatzort gehen.

\subsubsection{Versorgungsstrukturen und Preisniveau}

Bei den Akteuren vor Ort bestand die Befürchtung, dass durch die baustellenbedingten Zunahme der Bevölkerung und die damit einhergehende erhöhte Nachfrage das Preisniveau in der Gemeinde überdurchschnittlich steigen könnte. Aus Sicht einer nachhaltigen Entwicklung sollte im Verlauf der Baumassnahme ein wirt- 


\begin{tabular}{|l|l|l|}
\hline \multicolumn{1}{|c|}{ Ökologie } & \multicolumn{1}{|c|}{ Wirtschaft } & \multicolumn{1}{c|}{ Soziales } \\
\hline $\begin{array}{l}\text { Landschaft und Siedlungsbild } \\
\text { (Augenschein, } \\
\text { Flächennutzungsdaten) }\end{array}$ & $\begin{array}{l}\text { Versorgungsstruktur und Preisniveau } \\
\text { (Preiserhebungen) }\end{array}$ & $\begin{array}{l}\text { Stimmung in der Bevölkerung } \\
\text { (Meldungen am Beschwerdetelefon } \\
\text { und bei der Gemeinde) }\end{array}$ \\
\hline $\begin{array}{l}\text { Umweltmanagement } \\
\text { (Bericht Umweltbaubegleitung) }\end{array}$ & $\begin{array}{l}\text { Tourismus } \\
\text { (Informationszentrum, Baustellen- } \\
\text { bedingte Logiernächte) }\end{array}$ & $\begin{array}{l}\text { Integration der Bauarbeiter } \\
\text { (kulturelle Veranstaltungen und } \\
\text { Freizeitaktivitäten) }\end{array}$ \\
\hline $\begin{array}{l}\text { Naturhaushalt } \\
\text { (ökologischer Ausgleich, } \\
\text { Bericht Umweltbaubegleitung) }\end{array}$ & $\begin{array}{l}\text { Arbeitsmarkt } \\
\text { (Entwicklung Arbeitsplätze nach Art Anternehmen) }\end{array}$ & $\begin{array}{l}\text { Belästigungen durch die Baustelle } \\
\text { (Zahl der LKW-Fahrten, } \\
\text { Ausnahmebewilligungen } \\
\text { Strassenbeförderung) }\end{array}$ \\
\hline $\begin{array}{l}\text { Energieeinsatz } \\
\text { (Daten Ovra Electrica, } \\
\text { Gemeinde Tujetsch) }\end{array}$ & $\begin{array}{l}\text { Öffentlicher Haushalt } \\
\text { (Steuern, Investitionen der } \\
\text { offentlichen Hand) }\end{array}$ & $\begin{array}{l}\text { Verhältnis Gemeinde - Bevölkerung } \\
\text { (Beteiligung an } \\
\text { Informationsveranstaltungen) }\end{array}$ \\
\hline \multicolumn{2}{|c|}{ Integratives Kriterium } \\
\hline
\end{tabular}

Abb. 2: Kriterien zur Bewertung der Nachhaltigkeit des Bauprozesses (grau unterlegt: diese Kriterien werden im Folgenden vertieft behandelt)

Criteria and indicators to evaluate the sustainability of the construction process (highlighted in grey: criteria to be discussed in more detail later)

Critères et indicateurs pour évaluer la gestion durable du processus de construction (en gris: ces critères seront approfondis ci-dessous)

schaftliches und sozialverträgliches Preisniveau gehalten werden. Um den Einfluss der Baustelle auf das Preisniveau zu identifizieren, wurden die Preise ausgewählter Lebensmittel und Konsumgüter sowie die Preisentwicklung auf dem Immobilienmarkt erfasst. In Bezug auf Lebensmittel und Konsumgüter wurde schnell deutlich, dass sich die örtliche Preisentwicklung nicht wesentlich von der allgemeinen konjunkturellen Steigerung der Lebenshaltungskosten unterscheidet. Anders verläuft die Entwicklung auf dem Immobilienmarkt.

Bei der Entwicklung des Wohnungsbestandes hat Sedrun überdurchschnittliche Zuwächse zu verzeichnen, doch ist dies überwiegend durch den Bau privater Eigentumswohnungen verursacht und steht damit nicht in direktem Zusammenhang mit der Baumassnahme. Auch die Immobilienpreise sind bisher noch nicht gestiegen. Allerdings hat die Baumassnahme deutliche Auswirkungen auf das Angebot und das Preisniveau auf dem Mietwohnungsmarkt.

Die Beschäftigten der Baustelle wohnen grösstenteils in einer Containersiedlung unterhalb der Ortschaft.
Ein Teil der Beschäftigten - darunter mehrheitlich Kader-Angestellte - haben eine Wohnung in Sedrun oder Umgebung angemietet. Dies betrifft je nach Anzahl der Baustellenbeschäftigten zwischen 50 und 100 Wohnungen. Hierbei handelt es sich teils um Ferienwohnungen und teils um reine Mietwohnungen. Die Belegung der Mietwohnungen durch Baustellenbeschäftigte hat auf dem Mietwohnungsmarkt in Sedrun zu einer deutlichen Verknappung des Angebots und einem gestiegenen Preisniveau geführt. Etwa ein Viertel der von Baustellenbeschäftigten belegten Wohnungen liegt sogar ausserhalb Sedruns in einem Umkreis von bis zu einer Stunde Pendelentfernung.

Bei Gemeindeumfragen in den Jahren 2003 und 2007 wurde der Einfluss der Baustelle auf das Preisniveau von Seiten der Bevölkerung insgesamt aber grösstenteils als neutral bewertet (vgl. Abb. 4).

\subsubsection{Touristische Entwicklung}

Der Tourismus - insbesondere der Wintertourismus - ist für die Gemeinde Sedrun ein wichtiger Wirtschaftsfaktor. Obwohl sich Sedrun in den vergangenen 


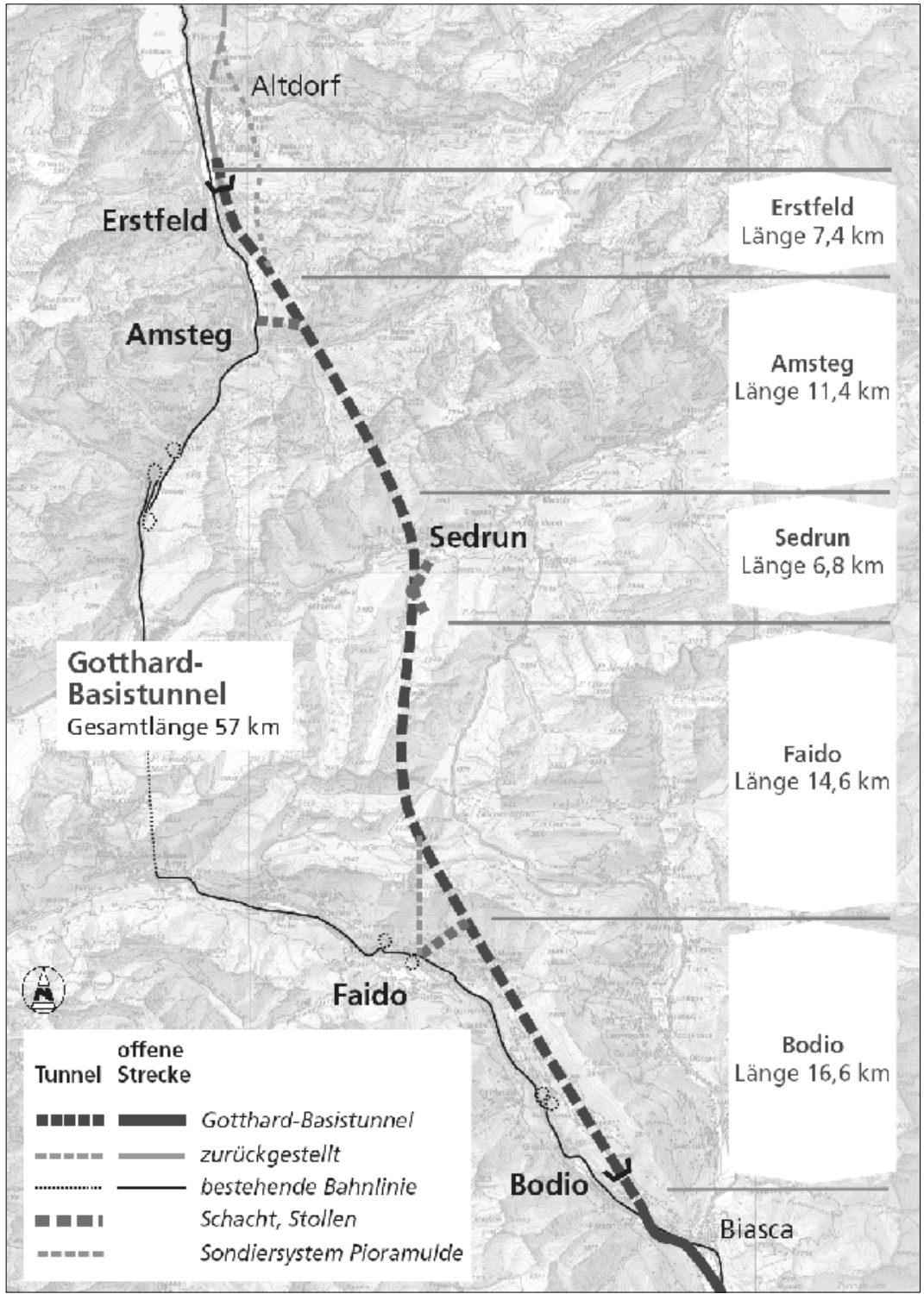

Abb. 3: Übersicht über die Linienführung des Gotthard-Basistunnels

Overview over the routing of the New Gotthard Base Tunnel

Aperçu du tracé de la ligne du tunnel de base du Saint-Gothard

Quelle: AlPTRAnsit GotTHARD AG (Abdruck mit freundlicher Genehmigung)

Jahren u.a. mit dem Tarifverbund mit den AndermattGotthard-Bahnen eine starke Position erarbeitet hat, sind auch hier die Auswirkungen des Strukturwandels in der Tourismusbranche zu spüren. Die Übernachtungszahlen sind in den vergangenen zehn Jahren stark gesunken, besonders die Ferienwohnungen hatten starke Einbussen zu verzeichnen.

$\mathrm{Zu}$ Beginn der Baumassnahme wurde das Ziel formuliert, dass die Baustelle den Tourismus nicht stören 


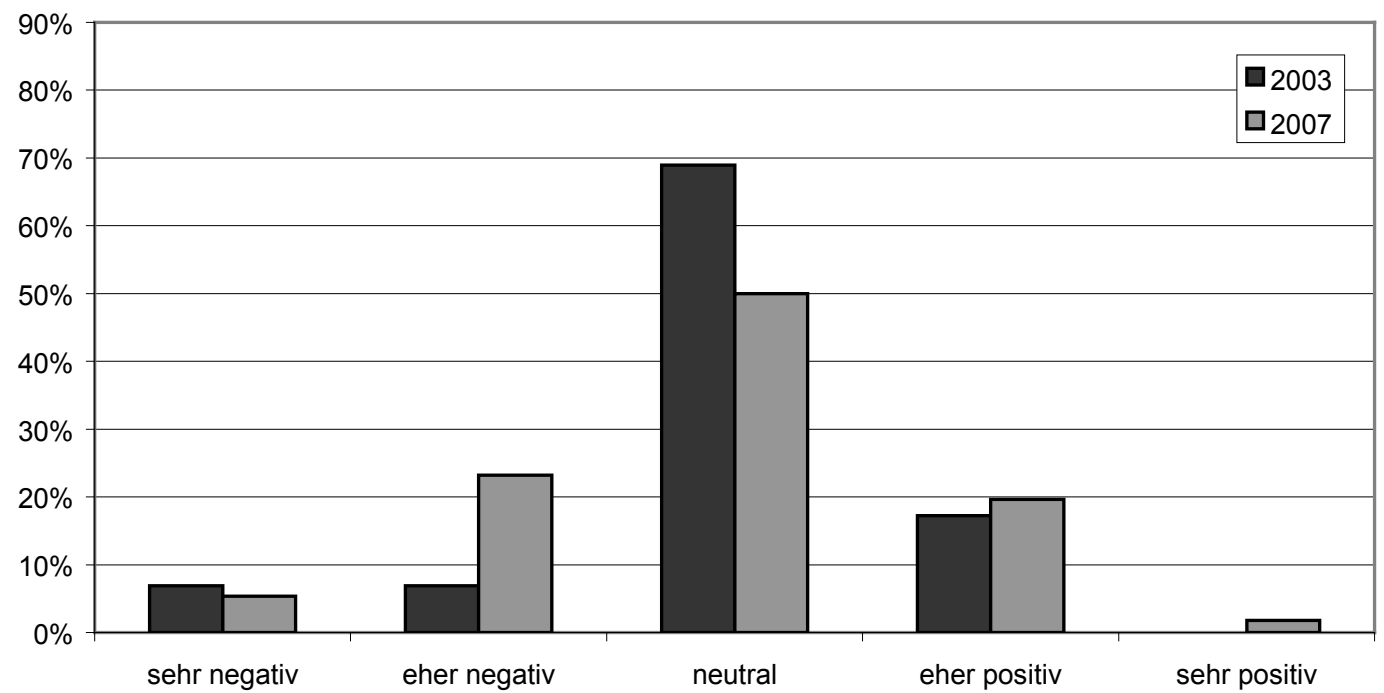

Abb. 4: Einfluss der Baustelle auf das Preisniveau in der Region (Einschätzung der Bevölkerung)

The influence of the construction site on price levels in the region (estimate of the locals)

Influence du chantier sur le niveau des prix dans la région (appréciation de la population)

Quelle: Strauf, Walser \& Bieger 2007

und vor allem in den Randzeiten zusätzliche Gäste in die Gemeinde bringen soll. Als Hintergrund dieser Überlegung gab es Befürchtungen, dass die Baustelle durch Lärm, Staub und Veränderungen des Landschaftsbildes einen negativen Einfluss auf die Attraktivität der Ferienregion haben könnte, und dass eine Konkurrenz der Feriengäste und der Baustellenbeschäftigten um die Ferienwohnungen und Gästebetten entstehen könnte. Auf der anderen Seite wurde die Baustelle aber auch als Chance gesehen, mit einem «Baustellen-Tourismus» zusätzliche Gästegruppen nach Sedrun zu locken. Der Einfluss der Baustelle auf den Tourismus war zu Beginn der Baumassnahme ein wichtiges Thema, das in Sedrun teilweise sehr emotional diskutiert wurde (vgl. Walser, Strauf \& Bieger 2004).

Zwar sind die Logiernächtezahlen insbesondere bei den Ferienwohnungen nach wie vor rückläufig, doch konnte die Hotellerie in den vergangenen fünf Jahren leichte Gewinne verbuchen (vgl. SEdrun Disentis Tourismus 2006). Ein Zusammenhang mit der Baustelle lässt sich in Bezug auf diese Entwicklung jedoch nicht herstellen, vielmehr zeigen sich ähnliche Entwicklungen in vielen vergleichbaren Alpendestinationen. Angesichts der Belegungszahlen kann man davon ausgehen, dass von einer Konkurrenzsituation zwischen Feriengästen und
Baustellenbeschäftigten allenfalls in der Hauptsaison, d.h. in wenigen Wochen im Winter, gesprochen werden kann.

Dagegen konnte mit Hilfe des Besucherinformationszentrums die Zahl der Tagesgäste deutlich erhöht werden. Die Ausstellung im Besucherzentrum verzeichnet eine starke Nachfrage, Baustellenführungen und Schachtbefahrungen sind derzeit mindestens ein Jahr im Voraus ausgebucht. Von den Tagesgästen profitiert insbesondere die Gastronomie.

Mittlerweile überwiegt auch in der Bevölkerung die Meinung, die Baustelle habe einen positiven Einfluss auf den Tourismus in der Region. Dies zeigen die Ergebnisse in den Gemeindeumfragen 2003 und 2007 (vgl. Abb. 5). Während 2003 noch knapp ein Drittel der Befragten sehr negative oder negative Auswirkungen der Baustelle auf den Tourismus wahrnahm, waren es 2007 nur noch 5\%. Dabei spielt sicherlich auch das starke Medienecho der Baustelle in Sedrun eine wichtige Rolle.

Eine ähnliche Tendenz zeigt sich auch in der Beurteilung der Konkurrenz um Ferienwohnungen: während 2003 noch $77 \%$ der Befragten eine starke oder sehr starke Konkurrenz sahen, waren dies 2007 nur noch $58 \%$. 


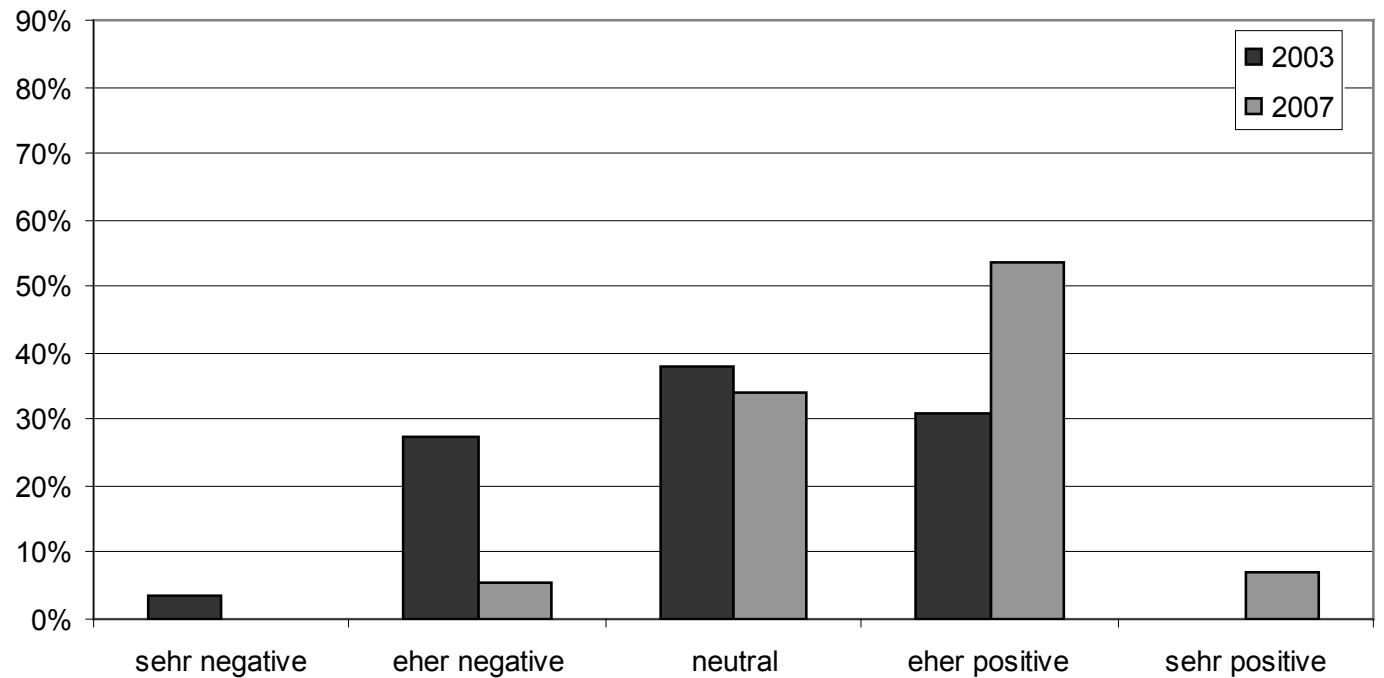

Abb. 5: Auswirkungen der Baustelle auf den Tourismus in der Region (Einschätzung der Bevölkerung)

The impact of the construction site on tourism in the region (perspective of the locals)

Conséquences du chantier sur le tourisme dans la région (appréciation de la population)

Quelle: Strauf, Walser \& Bieger 2007

\subsubsection{Bevölkerungsentwicklung und öffentlicher Haushalt}

Sedrun zählte im Jahr 1996 etwa 1'500 Einwohner, die Bevölkerung war in den Jahren vor Baubeginn relativ konstant und kaum von Abwanderungen betroffen. Im Vorfeld der Baumassnahme wurde erwartet, dass sich zeitweise bis zu 1'000 Baustellenbeschäftigte in der Gemeinde Sedrun aufhalten würden, und dass dies nicht nur auf die soziale Struktur in der Gemeinde, sondern auch auf die benötigte Infrastruktur einen grossen Einfluss haben würde. Im Laufe der Baumassnahme wurde jedoch deutlich, dass die Zahl der Baustellenbeschäftigten mit max. 700 Personen unter den Erwartungen bleiben würde. Da die Gemeinde ein Interesse daran hat, dass möglichst viele Beschäftigte während ihres Aufenthalts ihren Wohnsitz nach Sedrun verlegen, konnte die Zahl der in Sedrun mit Hauptwohnsitz gemeldeten Personen parallel zur Entwicklung der Beschäftigtenzahl auf der Baustelle in den letzten Jahren deutlich erhöht werden (vgl. Abb. $6)$.

Der Anstieg der Bevölkerungszahl hat direkten Einfluss auf die Gemeindefinanzen. Mit der Erhöhung der Einwohnerzahl sind die Einkünfte der Gemeinde aus Steuern natürlicher Personen und der Quellensteuer deutlich gestiegen. Diese beiden Steuern sind derzeit die Haupteinnahmequellen der Gemeinde (vgl. Abb. 7).
Mit dem Rückgang der Beschäftigten ab 2008 wird auch die Einwohnerzahl vermutlich bis zum Ende der Baumassnahme wieder auf das Niveau vor Baubeginn sinken. Durch die Unterbringung der Bauarbeiter in einer temporären Containeranlage halten sich die Investitionen der Gemeinde in Grenzen. Andere baustellenbedingte Investitionen (insbesondere zur Verkehrserschliessung) können nach Abschluss der Baumassnahme von der Gemeinde genutzt werden, wurden aber im Rahmen der Baumassnahmen finanziert. Hier zeigen sich positive Effekte der Grossbaustelle auf die Gemeinde und Region, die über die Bauzeit hinaus wirken.

\subsection{Soziale Auswirkungen}

Auf den ersten Blick ist die Baustelle von der Gemeinde aus kaum sichtbar. Der Installationsplatz der Baustelle sowie die Containersiedlung für die Beschäftigten befinden sich am Ortsrand etwas unterhalb der Gemeinde. Die Zusammensetzung der Arbeiterschaft ist international, insgesamt waren 2006 $14 \%$ der Beschäftigten Schweizer und 86\% Ausländer (Alptransit GotThaRd AG 2007). Etwa 70\% der Beschäftigten sind mit Wohnsitz in Sedrun gemeldet, knapp $19 \%$ wurden als Wochenaufenthalter geführt, gut $10 \%$ hatten ihren Wohnsitz auswärts (Auskunft Gemeinde TuJetsch 2007). Insgesamt ist die Fluktuation auf der Baustelle recht gering, die Beschäftigten 


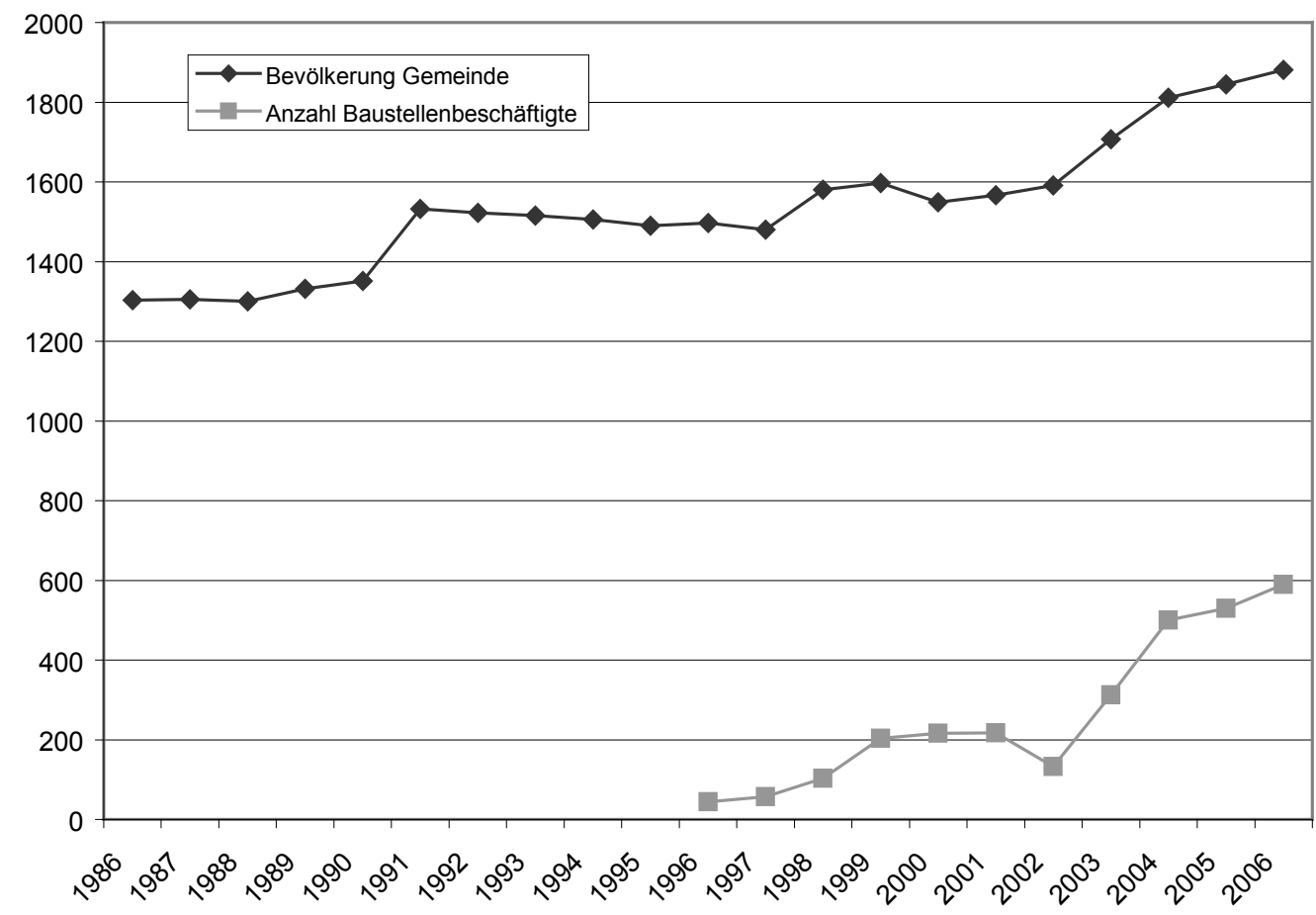

Abb. 6: Bevölkerungsentwicklung und Anzahl Baustellenbeschäftige

Population development and number of construction site employees

Evolution de la population et du nombre d'employés sur le chantier

Quelle: Amt FÜR Wirtschaft Und Tourismus DEs Kantons Graubünden (2007); eigene Darstellung

nehmen es als Privileg wahr, an diesem prestigeträchtigen Projekt mitarbeiten zu dürfen (Auskunft ARGE Transco Sedrun 2007).

\subsubsection{Belästigungen durch die Baustelle und Zustimmungsgrad der Bevölkerung}

Ein wichtiger und direkt messbarer Indikator für die Lebensqualität während der Bauphase ist der Grad der Zustimmung der Bevölkerung zur Baustelle. Vor Beginn der Baumassnahme gab es Unsicherheit und Ungewissheit, wie die Folgen der Baustelle aussehen könnten, speziell betreffend Ferienwohnungen, Hotels, Baustellenzufahrt und Immissionen (Staub, Lärm Geruch). Es gab im Vorfeld insgesamt 56 Einsprachen mit darauf folgenden Einigungsverhandlungen. Um den Anliegen der Bevölkerung Rechnung zu tragen, wurde sowohl bei der Gemeinde als auch beim Bauträger ein sogenanntes Sorgentelefon eingerichtet. Zu Beginn der Baumassnahme gingen 20 Meldungen ein, davon betraf die Hälfte Lärmentwicklungen. Bis Ende 2006 waren es insgesamt 56 Meldungen, wobei die Bevölkerung seit 1999 das
Angebot nur noch vereinzelt nutzt. Insgesamt werden die Belästigungen durch die Baustelle in der Bevölkerung als gering angesehen. $98 \%$ fühlen sich durch die Baustelle gar nicht oder eher wenig gestört (STRAUF, WALSER \& BIEgER 2007).

Die Einstellung der Bevölkerung gegenüber der Baustelle hat sich in den letzten Jahren nicht grundlegend gewandelt. Auch wenn es im Vorfeld Einsprachen gab, so sah doch bereits im Jahr 2000 die Mehrheit der Befragten die Vorteile für die Gemeinde - so die Ergebnisse einer Strassenumfrage. Diese Einschätzung besteht bis heute, in der Bevölkerung herrscht eine breite Zustimmung zur Baustelle. Auf die Frage, wer von der Baustelle profitiert, antworteten $98 \%$, dass die meisten oder alle einen Vorteil von der Baustelle haben (Strauf, Walser \& Bieger 2007). Als Hauptnutzniesser wurden genannt:

- Gastronomie/Hotellerie

- Gemeinde

- Bauwirtschaft/Baufirmen

- Einzelhandel und Gewerbe. 


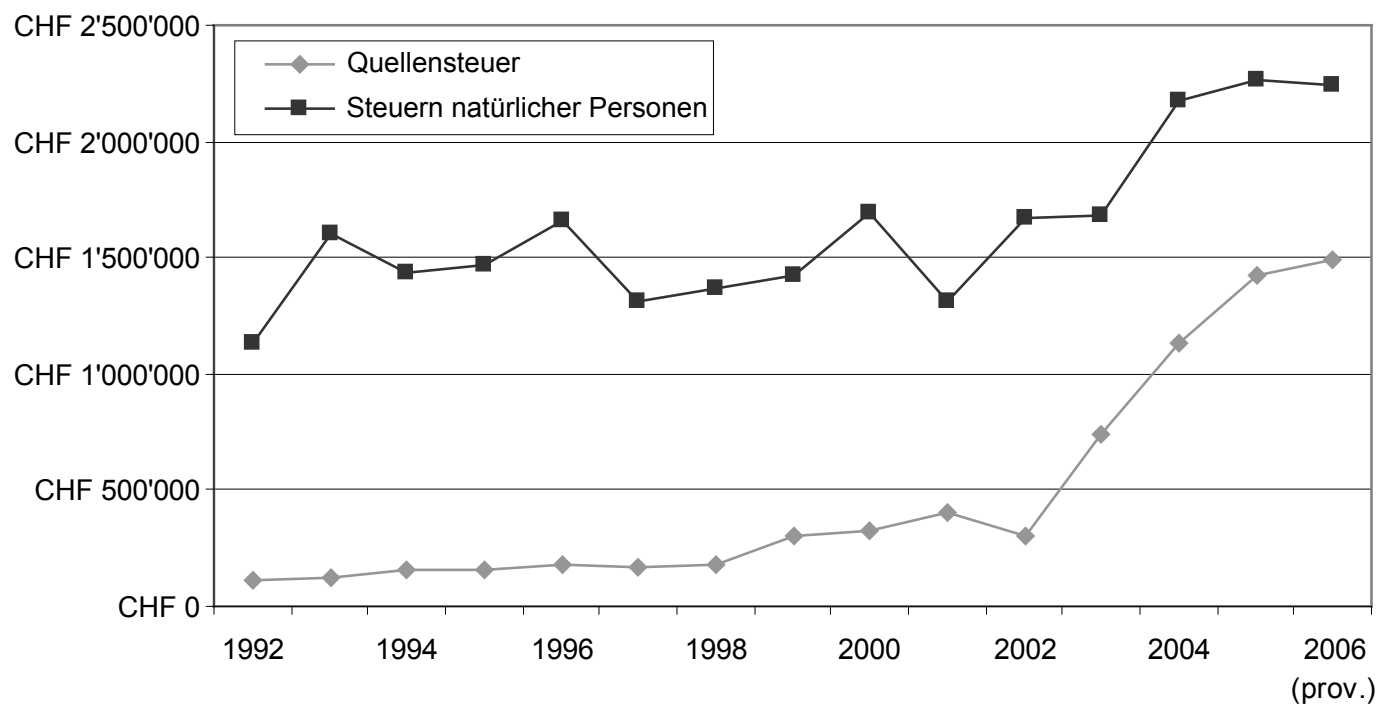

Abb. 7: Entwicklung der Steuereinnahmen der Gemeinde

Development of the community's tax revenue

Evolution des recettes fiscales de la commune

Quelle: GemeInde TuJETSCH; eigene Darstellung

\subsubsection{Integration der Baustellenbeschäftigten}

Bei den Überlegungen, welche Auswirkungen die Baustelle auf die Gemeinde hat, spielte nicht nur die für die Gemeinde sehr grosse Anzahl zusätzlicher Einwohner eine Rolle, sondern auch, ob und wie die Beschäftigten als Einwohner auf Zeit in das Gemeindeleben integriert werden können. Bei den Beschäftigten - insbesondere den Mineuren und Arbeitern - handelt es sich zum grossen Teil um ausländische Arbeitskräfte, die weder die deutsche noch die romanische Sprache beherrschen. Das Sprachproblem, die Angst vor Überfremdung und die Unsicherheit im Umgang mit ausländischen Kulturen waren - wenn auch nicht offiziell - in der Bevölkerung der Gemeinde ein Diskussionspunkt.

Frühere Überlegungen gingen auch davon aus, dass die Beschäftigten der Baustelle aktiv an Freizeitangeboten in der Gemeinde teilnehmen und die vorhandene Infrastruktur nutzen würden. Es wurde sogar überlegt, eigene Infrastrukturen oder Veranstaltungen für die Baustellenbeschäftigten einzurichten. Es wurde jedoch schnell deutlich, dass die Arbeitsanforderungen und das Leben auf der Baustelle den Beschäftigten wenig Freiraum für Freizeitaktivitäten lassen.

An die Beschäftigten stellt das Leben auf der Baustelle hohe Anforderungen: sie sind von ihrer Familie getrennt und haben nur alle 10-14 Tage Gele- genheit zur Heimfahrt. Es wird im 3-Schicht-Betrieb gearbeitet, die Arbeit als Mineur ist körperlich sehr anstrengend, und es bleibt wenig Zeit und Energie für Freizeitaktivitäten. Es herrschen Freizeitaktivitäten innerhalb der Containersiedlung vor (z.B. Fernsehen, Grillieren), Aktivitäten ausserhalb beschränken sich überwiegend auf den Besuch weniger Gaststätten, eigens organisierte Kulturveranstaltungen stiessen nur auf schwache Resonanz. Von Seiten der Belegschaft ist das Bedürfnis nach einer aktiven Gestaltung ihrer Freizeit sehr gering, vielmehr wird jede Gelegenheit zur Heimfahrt genutzt. Dies führt dazu, dass die Mehrzahl der Mineure und Arbeiter nur selten oder gelegentlich Kontakt zur einheimischen Bevölkerung hat.

Die Kader-Angestellten, die ihre Wohnung meist in der Gemeinde haben, sind relativ gut integriert. Von beiden Seiten wird das Verhältnis als unproblematisch beschrieben. Von Seiten der Bevölkerung werden die Baustellenbeschäftigten insgesamt als ruhig, angenehm und freundlich erlebt, die Befragten haben überwiegend positive Erfahrungen im Umgang mit der Belegschaft der Baustelle gemacht.

Diese eher unspektakuläre Einschätzung ist sicherlich auch auf die Situation Sedruns als Fremdenverkehrsort zurück zu führen. Den maximal 700 am Ortsrand eher «versteckten» Bauarbeitern stehen im Winterhalbjahr im Tagesdurchschnitt ca. 1'800 Skifahrer gegenüber 
(vgl. Sedrun Bergbahnen 2006). Der Anblick Ortsfremder ist also für Bevölkerung ein gewohnter.

\begin{abstract}
4.4 Weitere Auswirkungen auf die Gemeinde Sedrun Die Baustelle hat auch auf das soziale und gesellschaftliche Leben der Gemeinde in den letzten Jahren einen positiven Effekt gehabt. Die Identität und das Selbstvertrauen der Bevölkerung sind gewachsen. Durch die Baustelle wurde eine Dynamik ausgelöst, die Raum schafft für Visionen und Pläne, die ohne die Baustelle nicht entstanden wären. Insbesondere wäre hier die «Vision Porta Alpina» zu nennen, die mit grosser Mehrheit von der Gemeinde und der Region unterstützt wird und $\mathrm{zu}$ einem Identifikationspunkt geworden ist. Mit der Vision Porta Alpina ist die Hoffnung verbunden, durch eine bessere Erreichbarkeit die wirtschaftliche Zukunftsperspektive der Region deutlich zu verbessern (vgl. www.visiun-portaalpina. ch).
\end{abstract}

Darüber hinaus erarbeitete die Gemeinde im Zusammenhang mit der Tunnelbaustelle ein Entwicklungskonzept für die Gemeinde und ist inzwischen im Rahmen des Projektes PREGO (Projekt Raum- und Regionalentwicklung Gotthard) in eine überregionale und überkantonale Zusammenarbeit eingebunden. Dieses Projekt setzt sich mit der Zukunft des Gotthard-Raumes auseinander und versucht, die Vision eines lebendigen, einzigartigen Gotthard-Raumes, der attraktiv für seine Bevölkerung, die Wirtschaft und seine Gäste ist, umzusetzen (vgl. REgIERUNGSRAT Kanton UrI et al. 2007).

Jetzt steht die Gemeinde vor der Herausforderung, die baustellenbedingte Dynamik und wirtschaftliche Leistungsfähigkeit zu nutzen und heute die Weichen für die Zeit nach der Baumassnahme zu stellen, um damit eine nachhaltige Entwicklung der Gemeinde und der Region zu sichern.

\section{Fazit}

Vor dem Hintergrund des normativen Konzepts der nachhaltigen Entwicklung wurde untersucht, welche Effekte im Zusammenhang mit der NEAT-Baustelle in Sedrun identifiziert werden können. Hierzu wurden die Auswirkungen der Baustelle auf die Gemeinde und Region anhand ausgewählter Kriterien betrachtet. Als Zwischenergebnis kann man feststellen, dass die Baustelle nach zehn Jahren Bauzeit fester Bestandteil des Gemeindealltags ist. Keine der zu Beginn der Baumassnahme geäusserten Ängste und Befürchtungen sind eingetroffen, im Gegenteil konnten sowohl die Bevölkerung als auch die lokalen und regionalen Unternehmen und die Gemeinde selbst bisher wirtschaftlich von der Baustelle profitieren.
Auch im Alltagsleben der Bevölkerung hinterlässt die Baustelle kaum Spuren - deutlich weniger jedenfalls als die Gäste, die zum Wandern und Skifahren kommen.

Betrachtet man die historischen Aufzeichnungen, so zeigen sich erstaunlich viele Parallelen, aber auch Unterschiede zur aktuellen Situation. Die Sorgen und Ängste der Bevölkerung einerseits und andererseits die Chance, von der Baustelle zu profitieren, waren früher und sind heute eng mit der Vorbereitungsphase in den betroffenen Gemeinden verbunden. Sowohl früher wie heute hat die Baustelle wirtschaftliche Auswirkungen auf die jeweilige Gemeinde. Unterschiede im sozialen Bereich zeigen sich aufgrund geänderter Rahmenbedingungen (Kultur und Freizeitverhalten, Mobilität, Kommunikationstechnologien). Sie führen dazu, dass heute die zugezogenen Arbeiter viel weniger in die Gemeinde integriert sind und sein wollen.

Trotzdem gibt es auch heute soziale Auswirkungen der Grossbaustelle, die über das Ende der Bauzeit hinaus reichen. Waren es früher die italienischen Familien, die in Brig und anderen Orten ansässig wurden, so sind es heute in Sedrun eher Visionen und Projekte, deren Auslöser die Baustelle war, und die langfristig die Lebensqualität in der Gemeinde und Region beeinflussen können.

Insgesamt hatte die Baumassnahme bisher positive Auswirkungen auf ihre Standortgemeinde. Dass die Bevölkerung der Baumassnahme anfangs kritisch gegenüber stand, zeigen die genannten Ängste und Befürchtungen. Um den Anliegen der Bevölkerung $\mathrm{zu}$ begegnen, wurde und wird immer noch ein hoher Planungs- und Kommunikationsaufwand von Seiten der Bauherrin und der Gemeinde betrieben, um Probleme konstruktiv zu lösen. Die intensive Kommunikation und Kooperation zwischen der Gemeinde und der Bauherrin haben dazu geführt, dass die NEAT-Baustelle bisher von der Bevölkerung und der Gemeinde als Erfolg angesehen wird. Um die derzeit noch positiven Effekte der Baustelle für die Gemeinde und die Region langfristig zu nutzen, müssen jetzt Anstrengungen unternommen werden, um sich auf die Zeit nach der Baustelle vorzubereiten. Mit dem Ende der Baumassnahme werden nicht nur die zusätzlichen Steuereinnahmen der Gemeinde sinken, sondern auch die Hotellerie und Gastronomie sowie der Einzelhandel werden weniger Umsätze generieren. Daher steht die Gemeinde jetzt vor der Herausforderung, die entstandene Dynamik und das gesteigerte Selbstbewusstsein der Bevölkerung zu nutzen und die durch die Baustelle erzielten zusätzlichen Einnahmen langfristig und nachhaltig zu investieren. 


\section{Literatur}

Alptransit GotThard AG (o.J.): Monatsberichte der Umweltbaubegleitung für die Jahre 2000-2006. - Luzern.

Alptransit Gotthard AG (2007): Unveröffentlichte projektbezogene Auswertungen. - Sedrun.

Amt FÜr WirTschaft UND TOURISMUS DES Kantons GraUBÜNDEN (2007): Graubünden in Zahlen. - Chur.

Auskunft Gemeinde Tujetsch (2007): Schriftliche Auskunft der Gemeinde Tujetsch, P. Berther, Gemeindepräsident, Februar 2007. - Tujetsch.

Auskunft ARGE Transco Sedrun (2007): Persönliche Kommunikation mit Fr. Jörg-Madone, ARGE Transco Sedrun, 7.2.2007. - Sedrun.

Cantina Transalpina (2007): Mitten aus dem Leben am Portal zum Tunnel. - http://www.cantina-transalpina.ch 25.6.2007.

FASNACHT, A. (2003): Auswirkungen auf Grenchen. In: Kultur-Historisches Museum Grenchen (Hrsg.) (2003): Tripoli - wohnen und leben mit italienischen Tunnelarbeitern. - Begleitheft zur gleichnamigen Ausstellung des Kultur-Historischen Museums Grenchen im Rahmen der Grenchner Wohntage 03. - Grenchen: 49-53.

Gemeinde Tujetsch (o.J.): Projektbezogene Auswertungen. - Sedrun.

Kultur-Historisches Museum Grenchen (Hrsg.) (2003): Tripoli - wohnen und leben mit italienischen Tunnelarbeitern. - Begleitheft zur gleichnamigen Ausstellung des Kultur-Historischen Museums Grenchen im Rahmen der Grenchner Wohntage 03. - Grenchen. Regierungsrat Kanton Uri, Consigliere di Stato Cantone Ticino, Staatsrat Kanton Wallis \& Regierungsrat Kanton Graubünden (2007): San Gottardo: Das Herz der Alpen im Zentrum Europas. - Projekt Raum- und Regionalentwicklung Gotthard (PREGO). - o.O.

Sedrun Bergbahnen (2006): Geschäftsbericht 2005/ 2006. - Sedrun.

Sedrun Disentis Tourismus (2006): Jahresbericht 2005/2006. - Disentis, Sedrun.

Strauf, S., Walser, M. \& T. Bieger (2004): Die wirtschaftlichen Auswirkungen der NEAT-Baustelle in Sedrun auf die Region Obere Surselva. - Projektbericht. - St.Gallen.

Strauf, S., Walser, M. \& T. Bieger (2007): NEATBegleitforschung Langzeitstudie Sedrun. - Zwischenbericht 2006. - St. Gallen.

VIASTORIa \& K.T. Elsasser (Hrsg.) (2007): Der direkte Weg in den Süden - Zürich: AS-Verlag.

Walser, M., Strauf, S. \& T. Bieger (2004): NEATBegleitforschung Langzeitstudie Sedrun. - Zwischenbericht 2003. - St. Gallen.

WALTER, R.W. (2003): Grenchens erstes Spital befand sich im Tripoli. - In: Kultur-Historisches Museum GrEnchen (Hrsg.) (2003): Tripoli - wohnen und leben mit italienischen Tunnelarbeitern. - Begleitheft zur gleichnamigen Ausstellung des Kultur-Historischen Museums Grenchen im Rahmen der Grenchener Wohntage 03. - Grenchen: 19-22.

\section{Zusammenfassung: Eine NEAT-Grossbaustelle und ihre sozioökonomischen Auswirkungen auf die Standortregion}

Der Tunnelbau hat in der Schweiz eine lange Tradition. Der Bau der Neuen Alpentransversalen (NEAT) ist technisch höchst anspruchsvoll und hat nicht nur nach der Fertigstellung, sondern bereits während der Bauphase Effekte auf die Standortgemeinden. Bisher war es meist nur rückblickend möglich, diese Auswirkungen zu analysieren. Im Rahmen einer begleitenden Langzeitstudie hat die Gemeinde Sedrun (Graubünden) die einmalige Gelegenheit, die Auswirkungen der NEAT-Baustelle auf die Gemeinde zeitnah zu analysieren. Hierbei wird untersucht, welche ökologischen, wirtschaftlichen und sozialen Auswirkungen die Grossbaustelle vor dem Hintergrund des Konzeptes der nachhaltigen Entwicklung auf die Gemeinde hat, und welchen langfristigen Nutzen sie der Gemeinde bringen kann. Im Ergebnis zeigt sich, dass sich die anfänglichen Ängste und Befürchtungen der Bevölkerung bisher nicht bewahrheitet haben, und dass sowohl die wirtschaftlichen als auch die sozialen Effekte positiv waren. Nun steht die Gemeinde vor der Herausforderung, die durch die Baustelle erzielten zusätzlichen Einnahmen zu nutzen und nachhaltig zu investieren. Darüber hinaus können die in der Gemeinde entstandene Dynamik und das gesteigerte Selbstbewusstsein der Bevölkerung langfristig positive Auswirkungen auf die regionale Entwicklung haben.

\section{Abstract: A large NEAT construction site and its local socio-economic impact}

Tunnel construction has a long tradition in Switzerland. The construction of the New Alptransit (NEAT) is not only linked with great technical challenges, but the site itself has and will affect its surroundings during and after construction. Usually, such effects are analysed in retrospect. Within the context of a long-term study of the NEAT project, Sedrun, a mountain community in the Grisons, was given a rare opportunity to analyse the impacts of the NEAT construction while they were happening. The focus of the research project is on the local ecological, economic and social effects of the construction site in view of sustainable development or long-term benefits. The results indicate that the initial concerns of the locals regarding a negative impact of the construction site did not materialise. Both economically and socially, the effects can be viewed positively. In fact, the community is faced with the challenge of investing the resulting additional income for sustainable projects. Furthermore, it is possible that the growing dynamic and the increased self-confidence of the local actors could lead to a longterm positive effect on regional development. 


\section{Résumé: Les grands travaux de la NLFA et leurs impacts socio-économiques régionaux}

La Suisse a une longue tradition dans la construction de tunnels. La construction de la nouvelle ligne ferroviaire à travers les Alpes (NLFA) est non seulement très exigeante techniquement, mais elle a également des effets sur la région traversée, bien que les travaux ne soient pas encore achevés. Jusqu'ici, il n'était souvent possible d'analyser ces répercussions que rétrospectivement. Dans le cadre d'une étude longitudinale, le site de Sedrun (Grisons) permet d'analyser les effets actuels du chantier de la NLFA sur la commune. Dans ce contexte, cet article étudie les répercussions écologiques, économiques et sociales de ce grand chantier sur Sedrun, en regard du concept de développement durable et analyse quels bénéfices à long terme ce chantier peut apporter à la commune. Les résultats montrent que les craintes initiales de la population ne se sont jusque-là pas réalisées, dans la mesure où les effets socio-économiques sont positifs. La commune se trouve maintenant face au défi d'utiliser à bon escient les rentrées d'argent supplémentaires générées par le chantier et d'investir durablement. Au-delà, la dynamique naissante et la confiance de la population dans le projet pourraient avoir un effet positif à long terme sur le développement de la région.

Geogr. M.A. Simone Strauf, Dipl.-Verw.Wiss. Manfred Walser, Institut für Öffentliche Dienstleistungen und Tourismus (IDT-HSG), Universität St. Gallen, Dufourstrasse 40a, CH-9000 St. Gallen, Schweiz.

e-mail:

simone.strauf@unisg.ch

manfred.walser@unisg.ch

Manuskripteingang/received/manuscrit entré le 2.7.2007

Annahme zum Druck/accepted for publication/accepté pour l'impression: 29.11.2007 